\title{
Trombocitopenia Grave Induzida por Fenofibrato
}

\section{Severe Thrombocitopenia Induced by Fenofibrate}

\author{
Ana CLARA $\triangle^{1}$, Tiago MARTINS ${ }^{1}$, Alexandra MARTINS ${ }^{1}$, Ana BAPTISTA ${ }^{1}$, Mário LÁZARO1 \\ Acta Med Port 2020 Jan;33(1):58-61 • https://doi.org/10.20344/amp.11354 \\ RESUMO \\ A trombocitopenia induzida por fármacos é uma entidade frequente na prática clínica. No entanto, pela sua gravidade torna-se imperativo \\ distinguir a trombocitopenia não-imune das formas imunomediadas potenci-almente ameaçadoras da vida. Os autores descrevem o \\ caso clínico raro de um homem de 79 anos que se apresentou com púrpura trombocitopénica grave não-trombótica e gengivorragia \\ ao sexto dia de introdu-ção diária de fenofibrato na sua medicação habitual. Foi feita exclusão do fármaco e administrada metil- \\ prednisolona $125 \mathrm{mg}$ endovenoso durante três dias com resolução completa do quadro estabelecendo uma probabilidade elevada \\ de diagnóstico. A trombocitopenia associada ao fármaco é reportada pelos fabricantes como um evento extremamente raro. Este é o \\ segundo caso reportado de trombocitopenia imune ao fenofibrato, tendo o primeiro caso sido publicado em 2015. \\ Palavras-chave: Fenofibrato/efeitos adversos; Púrpura Trombocitopénica Idiopática/induzida quimicamente
}

\section{ABSTRACT}

Drug-induced thrombocytopenia is a common entity in clinical practice. However, having in consideration the severity of the case, it becomes imperative to distinguish non-immune thrombocytopenia from the po-tentially life-threatening immune-mediated forms. The authors report a rare clinical case of a 79-year-old man presenting with purpuric rash and gingival hemorrhage while on fenofibrate treatment (sixth day). The evolu-tion was favorable after drug removal and corticosteroid administration. Drug-associated thrombocytopenia is reported by manufacturers as an extremely rare event. This is the second case report of immune throm-bocytopenia to fenofibrate. The first event was reported for publication in 2015.

Keywords: Fenofibrate/adverse effects; Purpura, Thrombocytopenic, Idiopathic/chemically induced

\section{INTRODUÇÃO}

A trombocitopenia induzida por fármacos é uma entidade clínica que é tão frequente como é difícil o seu diagnóstico e abordagem. São vários os fármacos que podem levar a trombocitopenia com resposta imune ou não-imune. ${ }^{1} \mathrm{Ha}-$ bitualmente a forma não-imune apresenta um curso indolente. Por outro lado, a trombocitopenia imune induzida por fármacos (TIIF), corresponde a uma reação idiossincrática associada a um número mais limitado de fármacos e aparecimento de trombocitopenia grave e hemorragia 5 - 10 dias após introdução do novo medicamento. ${ }^{1,2}$

O fenofibrato é um derivado do ácido fíbrico com efeito modificador dos lípidos que através da ativação do recetor ativador dos peroxissomas alfa (PPARa) leva a: i) um aumento da lipólise e à eliminação plasmática de partículas aterogénicas ricas em triglicéridos; ii) aumento da síntese de apoproteínas Al e All; iii) redução das frações de VLDL e de LDL; iv) aumento da HDL. É globalmente seguro e os efeitos secundários prendem-se, sobretudo, com sintomas músculo-esqueléticos, gastrointestinais, elevação das transaminases e cefaleias. ${ }^{3}$

Apesar da trombocitopenia ser reportada enquanto efeito secundário extremamente raro durante a toma de fenofibrato, ${ }^{3}$ os autores apresentam um caso de púrpura trombocitopénica não trombótica que surgiu seis dias após o início da introdução de fenofibrato na medicação habitual. Considerou-se a hipótese diagnóstica de TIIF.

\section{CASO CLÍNICO}

Homem de 79 anos seguido no centro de saúde por diabetes mellitus tipo 2, hipercolesterolémia e hiperplasia benigna da próstata. Medicado há mais de quatro anos com sinvastatina $20 \mathrm{mg}$, metformina $500 \mathrm{mg}$, tansulosina $0,4 \mathrm{mg}$. Negou hábitos tabágicos e alcoólicos.

Um mês antes do internamento realizou análises que revelaram hipertrigliceridémia $(252 \mathrm{mg} / \mathrm{dL})$. Foi medicado 10 dias antes do internamento com fenofibrato $267 \mathrm{mg}$ um comprimido por dia. Ao sexto dia de toma referiu aparecimento de púrpura não pruriginosa, hematomas dispersos e gengivorragia. Realizou hemograma que revelou $16 \times 10 /$ $L^{\wedge 9}$ plaquetas tendo sido encaminhado ao serviço de urgência (SU). Não apresentou sinais e/ou sintomas de outro órgão ou sistema e negou toma de produtos de ervanária, viagens recentes e contacto com animais.

Ao exame objetivo apresentou púrpura não palpável nos membros inferiores até à raiz da coxa, não pruriginosas e não branqueáveis à digito-pressão; dois hematomas na fossa ilíaca esquerda e um no flanco esquerdo; dois hematomas no antebraço direito e três no antebraço esquerdo. Não apresentou adenomegálias palpáveis. Analiticamente, apresentava trombocitopenia grave de $17 \times 10 / \mathrm{L}^{\wedge 9}$ plaquetas e ausência de critérios de hemólise (Apêndice 1: https:// www.actamedicaportuguesa.com/revista/index.php/amp/ article/view/11354/Apendice_01.pdf). O esfregaço de sangue periférico demonstrou plaquetas grandes e ausência

1. Serviço de Medicina Interna. Centro Hospitalar Universitário do Algarve. Faro. Portugal.

$\bowtie$ Autor correspondente: Ana Clara. aritaclara@gmail.com

Recebido: 30 de setembro de 2018 - Aceite: 12 de novembro de 2019 | Copyright @ Ordem dos Médicos 2020 
de esquizócitos. Do restante estudo apresentou autoimunidade e serologias negativas. A electroforese de proteínas não apresentou alterações significativas (Fig. 1) Foi excluída esplenomegalia após realização de ecografia abdominal.

Foi considerado o diagnóstico de púrpura trombocitopénica iatrogénica com provável componente imune relacionada com a toma de fenofibrato. Realizou teste de ELISA para deteção de anticorpos contra antigénios HLA classe I e contra glicoproteínas plaquetárias IIb/IIla, la/Ila e Ib/IX: os resultados, conhecidos posteriormente, foram negativos.

Realizou exclusão do fármaco e iniciou três dias de administração de metilprednisolona $125 \mathrm{mg}$ endovenosa apresentando, à data da alta, lesões purpúricas (Fig. 2) e hematomas (Fig. 3) em fase de resolução com normalização do valor plaquetário (Apêndice 1). Foi avaliado em consulta externa, dois meses após alta hospitalar, e não apresentou quaisquer lesões cutâneas.

\section{DISCUSSÃO}

São vários os fármacos conhecidos que podem acelerar a destruição plaquetária através de mecanismos imunes ou não-imunes. ${ }^{4} \mathrm{Na}$ TIIF, as plaquetas tendem a ser afetadas por um processo de mediação imune com maior expressão na destruição plaquetária periférica. ${ }^{1}$ Esta entidade ocorre através da presença de anticorpos plaquetários com trombocitopenia moderada ou grave (plaquetas $<20 \times 10 / L^{\wedge 9}$ ) e hemorragia mucocutânea. Apresenta-se normalmente entre o quinto e $010^{\circ}$ dia após o início da exposição diária ao fármaco. ${ }^{1}$ Não existe um teste gold standard rápido e eficaz. Muitas vezes a confirmação laboratorial é tardia, sobretudo nos casos de hemorragia ameaçadora de vida. ${ }^{5}$ Apresenta uma fisiopatologia complexa tendo sido propostos pelo menos seis mecanismos específicos para diferentes fármacos, ${ }^{6}$ embora se desconheça, concretamente, qual é o mecanismo específico para o fenofibrato.

Por forma a estabelecer um diagnóstico provável entre trombocitopenia e um determinado fármaco foram propostos vários scores. ${ }^{6}$ Estes scores são, no entanto, pouco úteis perante a suspeita clínica de TIIF uma vez que só podem ser aplicados retrospetivamente. A confirmação prende-se, assim, com deteção in vitro de anticorpos anti-plaquetários dependentes do fármaco. ${ }^{5}$ Contudo, a implementação dos scores é dificultada pela ampla variedade de técnicas, pela falta de padronização e de validação dos mesmos. $^{7}$

Dimitrios et a $/^{\beta}$ publicaram, em 2015, o primeiro caso de trombocitopenia relacionada com o fenofibrato. Estes autores afirmam que o mecanismo mais provável será por destruição periférica e pela formação de anticorpos específicos contra o fenofibrato. Por sua vez, estes anticorpos reagem com as plaquetas através dos domínios Fab (fragment antigen-binding). No entanto, não foram realizados quaisquer testes confirmatórios da hipótese.

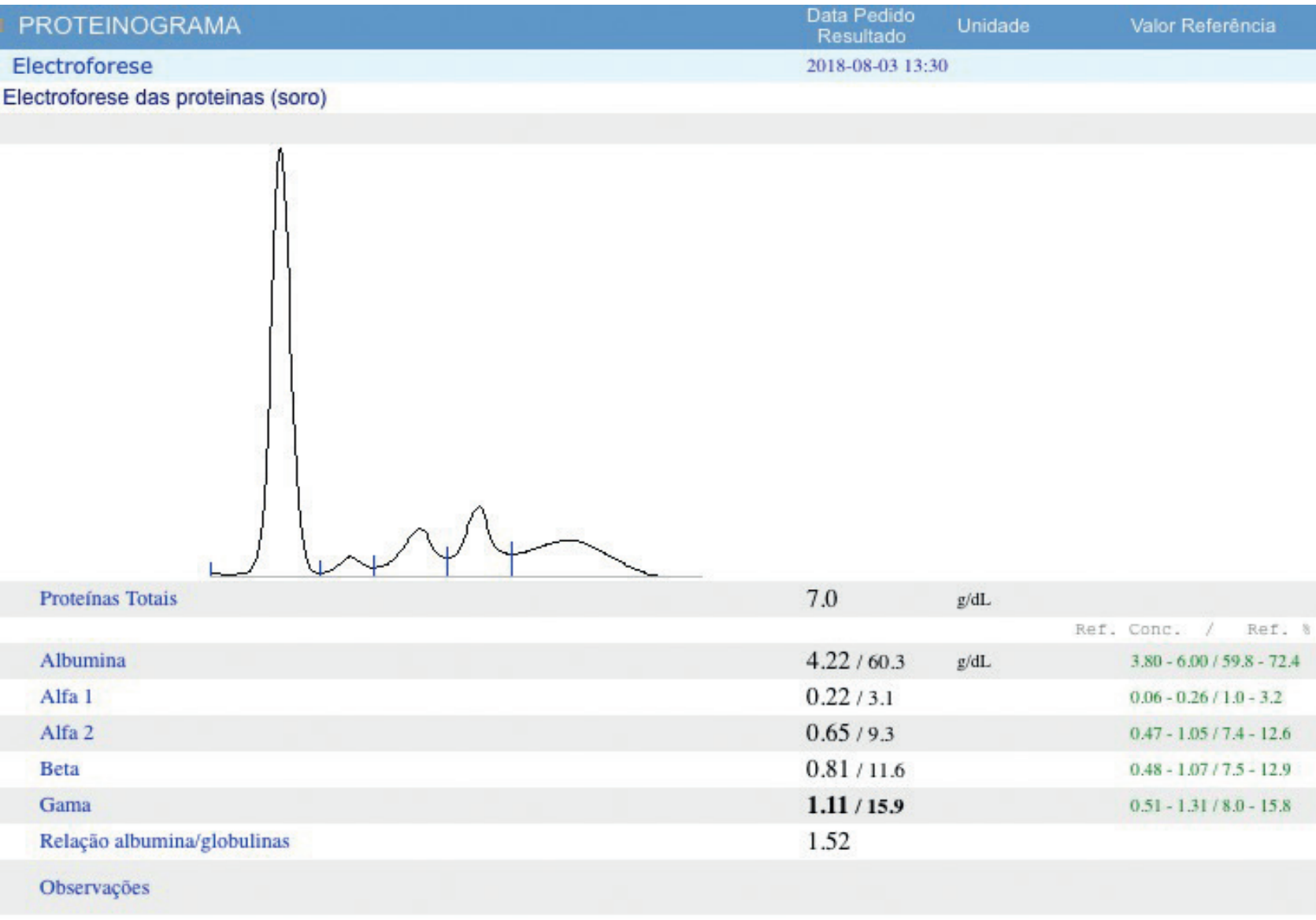

Figura 1 - Electroforese de proteínas 


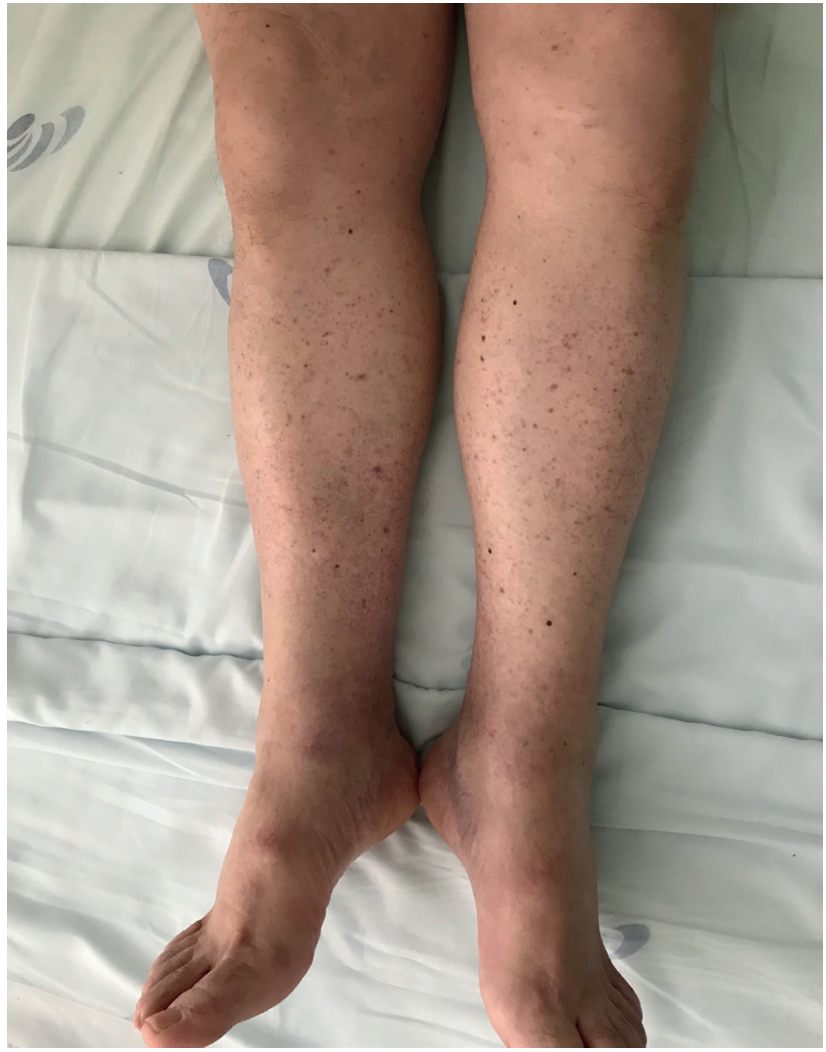

Figura 2 - Púrpura dos membros inferiores em fase de resolução

Não obstante a trombocitopenia ser um evento extremamente raro na toma de fenofibrato, no caso clínico apresentado parece haver uma forte correlação entre a introdução do fármaco e o início da sintomatologia assim como a sua resolução e não recorrência após exclusão do mesmo. O diagnóstico é apoiado pela ausência de outra condição que se poderia associar com trombocitopenia como hiperesplenismo, estados infeciosos, doenças autoimunes ou neoplásicas.

Deste modo estabelecemos como hipótese diagnóstica provável TIIF, perante a suspeita clínica apoiada nos seguintes pontos-chave: plaquetas $<20 \times 10 / L^{\wedge} 9$, hemorragia ativa e aparecimento dos sintomas 5 - 10 dias após introdução do fármaco. ${ }^{1}$

No caso reportado por Dimitrios et $a^{\beta}$ os sintomas surgiram apenas à terceira semana de exposição ao fármaco e foi necessária a administração de imunoglobulina e corticóides endovenosos durante um curso mais longo. Por outro lado, o caso clínico que os autores reportam enquadra-se nos critérios clínicos de diagnóstico, com os sintomas a surgir ao sexto dia de exposição. O tratamento baseou-se simplesmente na exclusão do fármaco e administração de três dias de metilprednisolona ev.

De referir que a confirmação do diagnóstico é suportada pela presença de anticorpos reativos em mais do que um teste laboratorial ${ }^{5}$ ou re-exposição ao fármaco. No caso clínico reportado foi realizada uma única pesquisa de anticorpos com resultados negativos e, por questões éticas, não

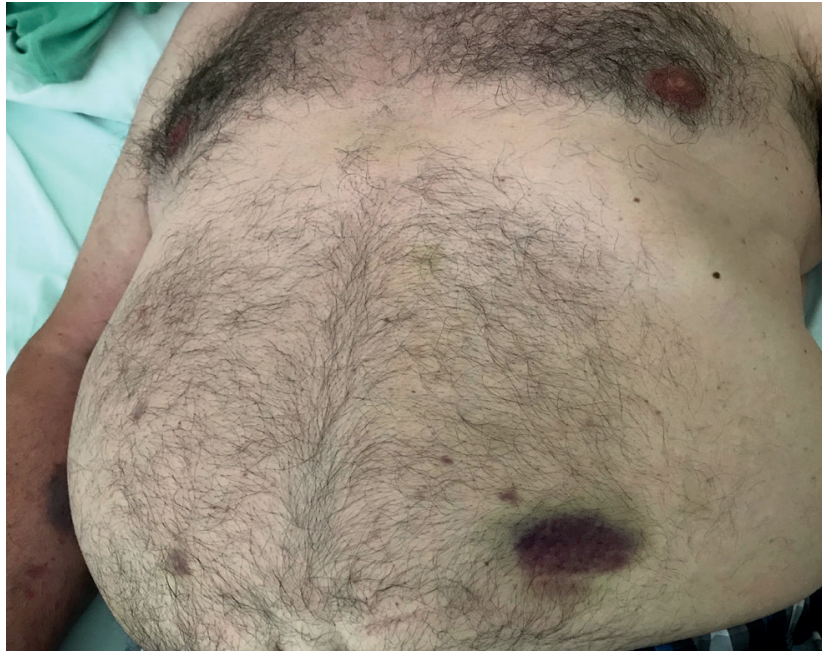

Figura 3 - Hematomas do abdómen e membro superior

foi realizada a re-exposição ao fármaco. Em Dimitrios et a/ ${ }^{8}$ não foram realizados testes confirmatórios e também não existiu re-exposição ao fenofibrato.

Pretendemos salientar a raridade do caso clínico apresentado. Perante a elevada suspeição clínica e trombocitopenia grave com hemorragia ativa torna-se imperativo o internamento para vigilância e monitorização. A exclusão do fármaco e introdução de corticoterapia durante três dias levou à normalização do número de plaquetas conferindo suporte à hipótese diagnóstica. Um único teste laboratorial negativo não exclui o diagnóstico. Consideramos ser fundamental o investimento na investigação laboratorial para desenvolvimento de testes específicos para o fenofibrato, tendo em conta a incidência anual de 10 casos por milhão de pessoas. ${ }^{9}$

\section{PROTECÇÃO DE PESSOAS E ANIMAIS}

Os autores declaram que os procedimentos seguidos estavam de acordo com os regulamentos estabelecidos pelos responsáveis da Comissão de Investigação Clínica e Ética e de acordo com a Declaração de Helsínquia da Associação Médica Mundial.

\section{CONFIDENCIALIDADE DOS DADOS}

Os autores declaram ter seguido os protocolos do seu centro de trabalho acerca da publicação de dados.

\section{CONSENTIMENTO INFORMADO}

Obtido.

\section{CONFLITOS DE INTERESSE}

Os autores declaram não ter qualquer conflito de interesse relativamente ao presente artigo.

\section{FONTES DE FINANCIAMENTO}

Não foi utilizada nenhuma bolsa ou subsídio para a realização do trabalho. 


\section{REFERÊNCIAS}

1. Arnold DM, Nazi I, Warkentin TE, Smith JW, Tolti LJ, George JN, et al. Approach to the diagnosis and management of drug-induced immune thrombocytopenia. Transfus Med Rev. 2013;27:137-45.

2. Kenney B, Stack G. Drug induced thrombocytopenia. Arch Pathol Lab Med. 2009;133:309-14.

3. FDA Adverse Events Reporting System (AERS). New Hampshire: Food and Drug Administration Website; 2008. [consultado 2018 set 29] Dlsponível em: https://www.accessdata.fda.gov/drugsatfda_docs/ label/2008/021350s008lbl.pdf.

4. De Silva E, Kim H. Focus on platelet apoptosis. Chem Biol Interac. 2018;284:1-11.

5. Arnold DM, Kukaswadia S, Nazi I, Esmail A, Dewar L, Smith JW, et al.
A systematic evaluation of laboratory testing for drug-induced immune thrombocytopenia. J Thromb Haemost. 2013;11:169-76.

6. George JN, Aster RH. Drug-induced thrombocytopenia: pathogenesis, evaluation, and management. Hematology Am Soc Hematol Educ Program. 2009:153-8.

7. Visentin GP, Liu CY. Drug induced thrombocytopenia. Hematol Oncol Clin of North Am. 2007;21:685-96.

8. Agapakis DI, Massa EV. A case of fenofibrate-induced immune thrombocytopenia: first report. P R Health Sci J. 2015;34:170-3.

9. Van den Bemt PM, Meyboom RH, Egberts AC. Drug-induced immune thrombocytopenia. Drug Saf. 2004;27:1243-52. 